\title{
ORDEN HÍDRICO: PRÁCTICAS E INSTITUCIONES EN MENDOZA, ARGENTINA
}

Paula Cecilia Mussetta pmussetta@mendoza-conicet.gob.ar Instituto de Ciencias Humanas Sociales y Ambientales

CCT-Conicet. Mendoza

\section{RESUMEN}

Este artículo aborda el campo del manejo de los recursos hídricos y propone un esquema de análisis para estudiar las relaciones entre las instituciones y las prácticas de los actores afectados por las acciones de tales instituciones. Avanza después, en forma preliminar y descriptiva, sobre la exploración de algunas de las categorías presentadas en el esquema propuesto en un caso concreto (Mendoza, Argentina). Reconocemos una doble dinámica de funcionamiento del régimen hídrico: una entre la autoridad hídrica y los grandes y poderosos actores vitícolas y otra entre un conjunto de entidades satélites no hídricas y pequeños productores. Esto lleva a que desde la política hídrica se reproduzca un modelo de producción que claramente beneficia a un sector a costa de otro.

Palabras clave: agua, instituciones, actores, desempeño.

HYDROLOGIC ORDER: PRACTICES AND INSTITUTIONS IN MENDOZA, ARGENTINA

ABSTRACT

This article addresses the territory of water resource management and proposes an analytical framework to study the inter-institutional relationships and practices of the stakeholders affected by the actions taken by those institutions. It explores in a preliminary and descriptive form some of the categories presented in the framework proposed for the specific case of Mendoza, Argentina. It identifies a double working dynamic in the hydrologic regime: one between the hydrologic authorities and large-scale and powerful stakeholders of the wine industry and another dynamic between a series of non-hydrologic satellite entities and small-scale producers. This leads to a hydrologic policy that reproduces a production model that clearly benefits one sector at the expense of another.

Key words: water, institutions, stakeholders, performance. 


\section{INTRODUCCIÓN}

Este artículo es un conjunto de notas de investigación, producto del avance de un proyecto orientado a conocer la relación entre el régimen institucional para la gestión del agua y el orden de prácticas hídricas locales en una región de tierras secas de la cuenca del río Mendoza, en el centro-oeste argentino.

Está organizado en tres partes. En primer lugar, expone y comenta el contexto político y académico actual marcado por esfuerzos orientados a fortalecer el control de los recursos hídricos; proponiendo como bandera explícita la participación de los actores en los procesos de toma de decisiones respecto del curso del agua, e implícita, una fe en las instituciones para intervenir los contextos locales y generar efectos deseados sobre el devenir hídrico.

La identificación de algunos puntos problemáticos en ese contexto es el inicio de la segunda parte. En ella se presenta un esquema de análisis (orden hídrico) que pretende superar algunas de las dificultades señaladas y entender otros aspectos que consideramos importantes en lo tocante a los actores: el desempeño de las instituciones y los contextos locales que estas alcanzan.

Tercero, avanza de manera preliminar y descriptiva sobre la exploración de algunas de las categorías presentadas en el esquema anterior en un caso concreto (Mendoza, Argentina), con el propósito de establecer algunos parámetros de articulación entre las instituciones, los actores y los contextos locales.

La investigación marco presenta un considerable grado de avance en las etapas de elaboración conceptual y de recolección de datos y un menor desarrollo de su análisis. ${ }^{1}$

De allí que estas páginas sólo presenten resultados preliminares, especialmente en la última parte. Aun así, es un valioso ejercicio cuyo principal aporte consiste en el conjunto de categorías y dimensiones para abordar los regímenes hídricos y que aquí decidimos llamar orden hídrico.

\footnotetext{
${ }^{1}$ El trabajo de campo consistió en más de 80 entrevistas en profundidad a actores institucionales y productores de la zona. En este trabajo sólo se presentan los resultados preliminares de las entrevistas a los actores institucionales.
}

\section{CONTEXTO DISCIPLINAR Y PUNTO DE PARTIDA}

El campo disciplinar de los recursos hídricos es demasiado amplio, heterogéneo y complejo. Por ello, la tarea de pretender encasillar posturas y conciliar ideas no es sólo imposible sino completamente improductiva. Ahora bien, no obstante las diferentes posiciones y maneras de definir las dificultades propias del sector -resulta casi innecesario aclarar que cuando se habla de agua en realidad se habla de problemas del agua- algunas afirmaciones han alcanzado un grado elemental de consenso.

Una de estas aseveraciones es que la problemática del agua no puede ser reducida a procesos naturales o físicos, ni obedece exclusivamente a leyes naturales o económicas, aun en regiones desérticas drásticamente marcadas por la escasez. Es decir, fenómenos que se definen a primera vista como acontecimientos naturales (escasez, sequías, inundaciones, efectos de eventos climáticos extremos) son procesos en gran medida social, política e incluso culturalmente construidos. A esto apuntan los enfoques integrales del agua que tanto auge tienen a la hora de definir planes y diseñar políticas.

Un elemento que se desprende de este consenso acerca del problema de la gestión hídrica es el que sostiene que gran parte de las debilidades de los regímenes es la inconsistencia entre las políticas y los problemas, entre las instituciones y las realidades a intervenir: muchos aspectos de esas realidades quedan fuera de la red de instituciones. Muchas instituciones son obsoletas para los problemas de las sociedades.

Ahora bien, este acuerdo/consenso sobre el problema de la gestión hídrica es tácito y generalizado: se adhieren una cantidad considerable de opiniones y corrientes diversas. Pero al mismo tiempo es muy concreto y específico: acaba en ese mismo enunciado y no se extiende a otro tipo de afirmaciones como, por ejemplo, al modo de resolver ese problema consensuado (la gestión antes que el recurso en sí). De hecho, justamente en ese punto se generan una cantidad de desacuerdos y miradas disonantes con las que queremos iniciar nuestra discusión.

Un nodo de fuerte disonancia se produce ante la definición de las reglas según las cuales los recursos hídricos serán consumidos, distribuidos, utilizados. Es decir, el consenso acaba en el mismo punto donde 
comienzan a discutirse las vías que ha de seguir el acceso y abastecimiento de agua. Esta decisión sobre el conjunto de instituciones que deberán organizar la gestión del recurso depende de procesos e historias sociales y políticas de cada lugar. No ocuparse de estas dimensiones menos naturales de los fenómenos hídrico-ambientales lleva a soslayar su complejidad y a obtener miradas sesgadas, poco útiles para resolver los problemas que afectan a nuestras regiones.

En un plano conceptual los paradigmas de la gobernanza y de los bienes comunes son dos de los modelos que actualmente alimentan gran parte de las discusiones. Ellos se nutren del problema de la crisis del modelo de gobierno estatal de los recursos naturales y de la necesidad de generar cambios relevantes. Estos dos paradigmas sustentan sus postulados en una amplia cantidad de experiencias exitosas de gestión hídrica basada en cierto tipo de diseño de principios institucionales (Ostrom 2008). Desde acá se preguntan ¿qué es lo que lleva a que un grupo de usuarios de un recurso logre un uso sustentable o, por el contrario, siga un patrón de uso en degradación?

La clave del éxito, sostienen, reside en el diseño de los principios institucionales con los que se rigen los actores y el recurso: el uso sustentable de un bien de uso común -como el agua- no solo es posible en condiciones diferentes a las de la coordinación central (es decir, coordinación por el Estado) y el mercado, sino que en ocasiones produce mejores resultados (Ostrom y Shlager 1992).

No vamos a referirnos en esta oportunidad a cada uno de ellos en extenso. Para los fines de este artículo basta con mencionar que el paradigma de la gobernanza, teórico y práctico, condensa en un tono normativo-prescriptivo el decaimiento de la autoridad regidora del Estado y el potenciamiento del mercado y la sociedad civil desde un plano de la ciencia política (Aguilar 2007). El de los bienes comunes es similar pero se concentra en fomentar la gestión en primer lugar y la propiedad colectiva en segundo, de los recursos o bienes de uso común. Uno y otro no son lo mismo porque tienen orígenes disciplinares y trayectorias conceptuales diferentes. Pero para el caso de los recursos hídricos pueden ser entendidos como dos versiones de una misma propuesta cuyo interés primordial se concentra en quitarle protagonismo a las instituciones públicas y estatales en la gestión del agua. En lo que sí interesa detenernos es en un problema que advertimos en la manera en que ellos conciben las instituciones.

\section{EL PROBLEMA CON LAS INSTITUCIONES Y LA PROPUESTA DEL ORDEN HÍDRICO}

Detengámonos un momento en asimilar cómo y por qué creemos que las instituciones entendidas así y en consecuencia las políticas y programas como sus instrumentos operativos, contribuyen a generar la problemática brecha señalada al inicio.

Estos enfoques contemplan las instituciones desde un punto de vista administrativo, exterior y objetivo a los actores sociales que las integran, como las estructuras que definen las interacciones humanas porque proveen el marco según el cual las personas deben actuar. Pero siempre son entendidas como algo externo a los actores, en ningún momento se tiene en cuenta su aspecto más subjetivo, esto es, que solo son tales en la medida en que los actores las actúan. Cuando se trata de recursos naturales, las instituciones son recursos de organización que se constituyen para cumplir una función específica en relación con ese recurso, esto sin considerar relaciones o estructuras sociales previas.

Completamente relacionado con el punto anterior, los esquemas propuestos no están preparados para reconocer (mucho menos incorporar) la complejidad de las situaciones que describen y pretenden intervenir. Asumen diferencias no interactivas o dinámicas entre lo formal y lo informal, lo local y lo global (Mosse 2006, Metha et al. 1999). En consecuencia, las prescripciones políticas se dirigen o bien al ámbito formal (recomendaciones para el Estado), o bien al de las instituciones locales, sin considerar la cantidad de situaciones en las que estos límites son borrosos. Esta dicotomía, al igual que la de local/ global deja fuera las realidades empíricas en las que predominan los cruces a los que nos referimos antes.

Y además, son consideradas entidades ahistóricas y estáticas en tanto los enfoques no analizan flujos de acciones sino fotografías de los estados de situación de un recurso particular. Los modelos que nos presentan consisten en una relación casi mecánica entre política, implementación y resultados. Pero no consideran que las instituciones se insertan en un campo social con una historia de acciones y relaciones sociales que no puede obviarse ni anularse como 
si no existiera. Y esto porque ven reglas antes que prácticas sociales.

Asumen una mirada romántica y optimista del concepto comunidad como una entidad homogénea y con capacidad para establecer relaciones vinculantes, sin atender las profundas e importantes diferencias sociales entre actores ni relaciones conflictivas o de poder entre ellos (Nuijten 2005). Algunos que sí llegan a verlo, igualmente niegan el papel explicativo de las dimensiones socioculturales de creencias y de información así como las asimetrías de poder. $\mathrm{Y}$ esto porque no pueden ver procesos que son completamente cualitativos como la jerarquía, el poder, las construcciones culturales, interpretaciones de los agentes. Es decir, los ven pero al tratar de cuantificarlos, los simplifican de tal forma que terminan por anularlos y quitarle importancia explicativa.

Reconocen un comportamiento racional de los actores sin ver la posible multiplicidad de interpretaciones. Por ello es que logran desarrollar modelos predictivos que describen las condiciones generales necesarias para amortiguar los efectos negativos del uso no sustentable de los recursos. Si bien este tipo de modelos resultan atractivos para los hacedores de políticas porque permiten establecer generalizaciones, creemos que justamente allí reside su debilidad (Mosse 2006, Birkenholtz 2011). Cuando estas generalizaciones bajan a la práctica se vuelven obsoletas.

Si las instituciones así entendidas no hacen más que reproducir la brecha entre las políticas y los problemas reales, ¿cómo conviene entenderlas? La cuadro 1 resume algunas ideas principales de la línea propuesta.

Cuadro 1. Dos miradas de las instituciones.
Los enfoques dominantes plantean panaceas institucionales para resolver los problemas de gestión hídrica y de equidad (cuadro 1). Estas instituciones tendrían la capacidad de interferir en las realidades sociales de manera programada y lineal. El pensamiento sostiene que basta con diseñar principios institucionales adecuados para lograr las metas propuestas.

Creemos en cambio que esta es una manera extremamente simplificada de entender el proceso de intervención institucional. Las comunidades no reaccionan de manera unilateral ante las reglas formales. Junto al orden institucional formal, los actores sociales a quienes se dirigen las instituciones desarrollan también sus propias estrategias, reglas y acuerdos locales para apropiarse del agua que necesitan para desarrollar sus actividades productivas. En la base de esta producción normativa local y en un contexto de tierras secas como Mendoza, Argentina, afectado además por eventos climáticos extremos, se encuentra una demanda de agua no satisfecha y una necesidad concreta de acceso al recurso.

El resultado es un orden hídrico local basado en transacciones y negociaciones de formalidad variada que convive y se relaciona -en oposición y/o negociación- con el orden político institucional formal o dominante. La normativa formal institucional configura y se entreteje en las comunidades locales, en la sociedad, y estas se organizan para hacer frente a las necesidades produciendo un orden propio de estrategias que a la vez generan otro tipo de institucionalidades.

Optamos por desarrollar otra mirada de ida y vuelta de relación entre las instituciones y la realidad social que, además, atienda los espacios intermedios, los

\section{INSTITUCIONES}

\section{Gobernanza y bienes comunes}

El producto de la voluntad política de los actores.

Los actores reaccionan a las reglas formales.

Existen dicotomías netas entre los aspectos formales y los informales de las instituciones.

Son las reglas y los recursos que orientan las acciones de los actores.
Mirada del orden hídrico

El producto de historias de luchas, negociaciones y competencias.

Junto a las reglas, desarrollan sus propias estrategias y acuerdos. Entre las instituciones formales y las realidades locales existen unos espacios intermedios que son más importantes para la gestión del recurso que las normas formales.

Son una compleja red de prácticas, arenas de interacción entre actores que expresan relaciones de poder y marcos culturales.

Fuente: Elaboración propia.

P. C. Mussetta • Orden hídrico: prácticas e instituciones

en Mendoza, Argentina 
intersticios entre uno y otro. A ello llamamos «orden hídrico»: es el producto que resulta de la vinculación entre las instituciones formales y las prácticas de los actores locales; en otras palabras, son los marcos institucionales -lo que ellos restringen y/o habilitan-, programas, políticas, mecanismos, etc., pasados por el tamiz de su reapropiación y transformación por sus actores destinatarios. En otras palabras, es el conjunto de las relaciones hidrosociales que surgen entre lo que las instituciones proponen y producen y lo que los actores hacen con ello. Ahora bien, en términos operativos, ¿qué y cómo estudiar del régimen hídrico para no caer en las miradas dominantes señaladas más arriba?

\section{LOS COMPONENTES DEL ORDEN HÍDRICO}

Figura 1. Los componentes del orden hídrico.

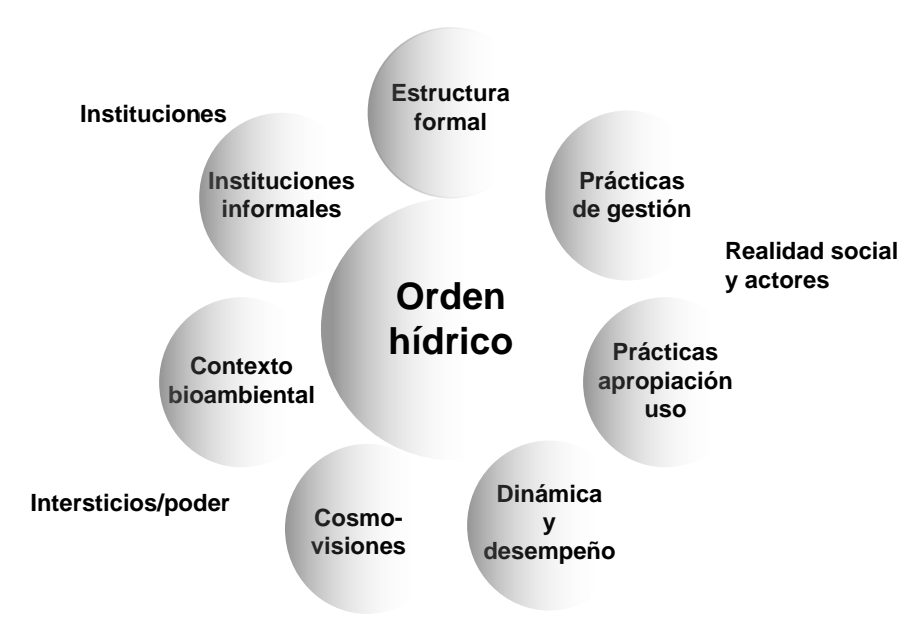

Prácticas de apropiación y uso: De igual modo que con los actores institucionales, las interacciones de los usuarios del agua demuestran que las estructuras son transformadas y reinterpretadas por los sujetos según ciertos parámetros que definen la situación del usuario.

Dinámica y performance: La dinámica y el desempeño se refieren a las interacciones horizontales y verticales entre las instituciones encargadas del agua. Esto nos dará información sobre la concentración y coordinación de la autoridad.

Cosmovisiones de los actores: Se refieren a los sentidos y valoraciones simbólicas que los actores otorgan a las instituciones, las percepciones sobre el régimen y sus actores. La importancia de estos aspectos «menos materiales» del orden hídrico es determinante porque aporta (o quita) la legitimidad necesaria para el sostenimiento de las instituciones formales e informales.

Contexto bioambiental: las características biofísicas del entorno son fundamentales porque marcan las condiciones más elementales del recurso hídrico. No obstante, estas condiciones naturales no son las determinantes exclusivas de las situación de los usuarios del agua ya que según sus capacidades y recursos cada actor podrá administrar esas condiciones naturales de manera diferente.

Instituciones informales: es el conjunto de reglas y códigos no escritos pero comúnmente conocidos que determinan la vida social y política de forma a veces más visibles que otras, creando

Fuente: Elaboración propia.

Estructura institucional formal: es el conjunto de rasgos de largo plazo estructurales e interdependientes que regulan el agua (leyes, normas jurídicas, estatutos, instituciones formales, políticas y programas).

Prácticas de gestión: son las acciones e interacciones concretas de los actores que integran las instituciones formales (funcionarios públicos). La relevancia de analizar las prácticas además de las instituciones se funda en una necesidad de darle prioridad a los actores ya que las estructuras formales no necesariamente coinciden con lo que hacen los actores encargados de implementarlas. o restringiendo los incentivos para cumplir las reglas formales (Helmke y Levitsky 2012). Además, desempeñan el papel de motivar el cumplimiento de las instituciones formales.

A continuación, nos referiremos a estas dimensiones para el caso de la cuenca del río Mendoza. En esta oportunidad solo nos referiremos a algunas.

\section{EL ORDEN HÍDRICO MENDOCINO}

Contexto bioambiental. La cuenca del Rio Mendoza está ubicada en el centro oeste de Argentina, en la latitud de los Andes centrales. Se encuentra en una región semidesértica cuya sequía y escasez hídrica condicionan las posibilidades de producción local, 
tanto agrícola como ganadera, y que solo sea posible la agricultura de riego. De esta forma el agua fragmenta el territorio en dos contextos claramente diferenciados: los oasis artificiales de riego por un lado y las zonas no irrigadas por otro.

Si bien la falta de agua es estructural e inherente al territorio, en los últimos años muestra un agravamiento por la presencia de efectos climatológicos adversos, principalmente heladas fuera de temporada, lluvia en momentos críticos para los cultivos, granizo y aumento de la temperatura. Esto ha llegado a generar pérdidas de $10 \%$ de la producción.

Estructura institucional formal. Señalaremos solo algunos aspectos puntuales, los que creemos que son relevantes para entender el tipo de inquietudes que nos planteamos.

Autonomía provincial sobre los recursos: Argentina no cuenta con una ley nacional de aguas y son las provincias (entidades federativas) las que tienen el dominio originario de los recursos hídricos, es decir, gozan de autonomía jurídica sobre el agua. De esta forma, en la provincia de Mendoza la única autoridad hídrica es el Departamento General de Irrigación, goza de autarquía y rango constitucional.

Cuencas y prioridad de usos: La cuenca como unidad de gestión es un concepto teórico que lejos está de ser aplicado en la práctica. Aunque en la letra formal se proyecta como un régimen de gestión integral que atiende a todos los usos, existe en la práctica una clara jerarquía del riego por sobre los demás usos. Esto responde a un modelo de desarrollo vitícola instaurado en el siglo XIX (año del que data la actual ley de aguas de la provincia): el agua que baja de la Cordillera de los Andes se almacena, se distribuye, se sistematiza en riego agrícola y acaba en la producción. Ese modelo orientado a un modelo vitícola dio forma a la administración del agua en Mendoza y hoy continúa. En ese momento fue diseñado por una elite planificadora con el poder político, social y económico de incidir en los destinos productivos de la región.

Agua para producción y no para ambiente. En completa relación con lo anterior, no solo en términos de jurisdicción formal, sino también en la práctica más cotidiana, el agua es competencia exclusiva del área productiva mas no de la ambiental. Las dependencias ambientales de los municipios y del mismo gobierno provincial se relacionan solo de manera indirecta con la gestión hídrica: las pri-

P. C. Mussetta • Orden hídrico: prácticas e instituciones en Mendoza, Argentina meras se ocupan del agua potable (abastecimiento y educación), las segundas principalmente de eventos climáticos.

Inherencia del agua a la tierra. Otro aspecto institucional formal importante por mencionar es el principio jurídico que indica que el agua le pertenece a la tierra en la que se encuentra. Pero además de la atadura al territorio, hay otra atadura al derecho por uso adquirido. Es decir, no sólo no es posible jurídicamente trasladar el agua de un territorio a otro, sino que no es posible modificar una dotación de agua si está asociada a un derecho de riego. La posición oficial (autoridad hídrica) sostiene que un derecho definitivo otorgado a un territorio para agricultura no puede cambiar su uso de suelo. ${ }^{2}$

Frente a esto, otros actores públicos, con menos poder de decisión, expresan la visión de eliminar la inherencia del agua a la tierra para promover el uso más eficiente del recurso de acuerdo al tipo de suelo y según el tipo de cultivo. Esto implica un enfoque orientado en la demanda del cultivo y no en la oferta como actualmente se da.

Prácticas de gestión: patrones de interacción entre usuarios e instituciones. Para entender las prácticas, previamente es necesario presentar una breve descripción de los actores y la estructura productiva (cuadro 2). ${ }^{3}$

Las interacciones que se establecen entre los usuarios del agua para riego y las instituciones no son iguales sino que varían según el tipo de productor rural y se da un desbalance en la atención de las necesidades de cada grupo.

Entre los actores que poseen derechos de riego, los grandes productores irrigados (columna 1, cuadro 2) mantienen otro patrón de interacción con las instituciones. Como sostiene Montaña (2013) su relación con el Estado es indirecta y pasa por la regulación del mercado, la política fiscal y la política exportadora. De esta forma alcanzan cierto grado de

${ }^{2}$ De hecho, hoy se propone llevar al extremo el cumplimiento de este principio expropiando tierras con derechos de agua otorgados que se encuentren en estado improductivo. Entrevista al secretario de Gestión Hídrica del Departamento General de Irrigación. Septiembre de 2012.

${ }^{3}$ Para la elaboración de los apartados que describen las prácticas de gestión y las de uso y apropiación fue fundamental el análisis previo realizado por Elma Montaña, publicados en Montaña (2012, 2013). 
independencia del sistema con la autoridad hídrica provincial e incluso con los financiadores. Un contexto institucional en que el poder de decisión sobre el agua está concentrado en una única autoridad, junto a un contexto bioambiental de escasez ubica a este grupo de actores en una posición de privilegio exclusivo para obtener la cantidad de agua que necesitan y más. En el mapa de las relaciones institucionales, el Departamento General de Irrigación parece operar y estar a disposición para un restringido sector de productores: los grandes productores estas solo son asociaciones de usuarios que funcionan como órganos consultivos no vinculantes al proceso de toma de decisiones. Por lo tanto, no es de aquí que pueden obtener respuestas a sus problemas.

Las interacciones más frecuentes con instituciones que este conjunto de productores establece son, en primer lugar, con las dependencias de producción y de ambiente (nacionales, provinciales), no hídricas. Es común que ellos reciban asistencia por contingencias climáticas antes que por agua. Por ejemplo, productores afectados por granizo son asistidos en forma directa con algunos insumos para levantar su producción con estrategias diversas: replantar en

vitícolas. Esto ilustra la afirmación previa sobre la perpetuidad del modelo de desarrollo vitícola instaurado por la elite en siglo XIX.

Cuadro 2. Características de la producción y sus actores.

\begin{tabular}{|c|c|c|c|}
\hline \multicolumn{3}{|c|}{ Productores de la zona irrigada } & \multirow{2}{*}{$\begin{array}{c}\text { Productores de la zona } \\
\text { no irrigada }\end{array}$} \\
\hline 1 Grandes productores & 2 Medianos productores & 3 Pequeños productores & \\
\hline $\begin{array}{l}\text { Modelos vitícolas } \\
\text { tecnificados. } \\
\text { Vinculado al sector } \\
\text { agroalimentario mundial. } \\
\text { Estructura altamente } \\
\text { organizada e } \\
\text { institucionalizada } \\
\text { Localizados en las partes } \\
\text { más favorecidas de la cuenca } \\
\text { (altas). }\end{array}$ & $\begin{array}{l}\text { Modelo vitícola tradicional. } \\
\text { Producto de menor calidad y } \\
\text { orientado al mercado interno. } \\
\text { Escasamente integrados al } \\
\text { eslabón industrial. } \\
\text { Alta economía informal } \\
\text { (en mano de obra y } \\
\text { transacciones). } \\
\text { Sin planificación a mediano y } \\
\text { largo plazo. }\end{array}$ & $\begin{array}{l}\text { Principalmente } \\
\text { horticultores. } \\
\text { Acuden a redes } \\
\text { familiares para } \\
\text { organizar su } \\
\text { producción. } \\
\text { Desconocimiento de } \\
\text { sus derechos. } \\
\text { Arriesgan su } \\
\text { supervivencia. }\end{array}$ & $\begin{array}{l}\text { Ubicados en la } \\
\text { parte más baja y } \\
\text { desfavorecida de la } \\
\text { cuenca. } \\
\text { Prácticas ganaderas } \\
\text { (caprina) de } \\
\text { subsistencia. } \\
\text { Complementan sus } \\
\text { ingresos con trabajos } \\
\text { temporales en la } \\
\text { agricultura. }\end{array}$ \\
\hline $\begin{array}{l}\text { Algunos horticultores, con } \\
\text { perfil menos empresarial que } \\
\text { los viticultores. } \\
\text { Menor utilización de } \\
\text { tecnología. } \\
\text { Planificación a mediano y } \\
\text { largo plazos. }\end{array}$ & $\begin{array}{l}\text { Horticultores con perfil } \\
\text { similar al de los pequeños. }\end{array}$ & & \\
\hline
\end{tabular}

Fuente: Elaboración propia con base en información presentada en Montaña 2013.

A diferencia de los grandes, los pequeños y medianos productores (columnas 2 y 3 de la cuadro 2) mantienen con la autoridad hídrica (Departamento General de Irrigación) una relación estrictamente formal, mediada por la posesión de títulos de riego. Este grupo de productores también tiene vínculos directos con las instancias intermedias de gestión hídrica (denominadas Inspecciones de Cauce), pero caso que se trate de un cultivo hortícola o pasar ese ciclo con un cultivo permanente, para que al siguiente puedan tener cierta competitividad. Respecto del riego reciben asistencia técnica muy específica también de dependencias de producción y clima en ahorro de agua intrafinca (asesorías sobre formas de riego, cuidado del agua y de los terrenos para eficientar lo disponible, aprovechamiento del tipo de frecuencias o de caudal de los terrenos de la mejor manera). También son asistidos en infraestructura, en obras 
de recuperación de perforaciones abandonadas y de canalización.

De esta forma, este grupo de actores tiene gran interacción con las agencias municipales de producción y desarrollo pero estas no tienen ni injerencia, ni jurisdicción, ni poder de decisión sobre los asuntos hídricos. Mucho menos recursos. Los municipios logran ser mediadores entre los actores locales y otras instancias para ayudarlos a acceder a programas de economía social y de sustento. Esta es una de las pocas opciones para aquellos que de manera independiente no pueden acceder a otros circuitos de comercialización o crédito.

Por último, los productores caprinos en la zona no irrigada (columna 4 de la cuadro 2) son los que se encuentran en peores condiciones. Ellos no tienen derechos adquiridos, por lo que se mantienen fuera de la órbita de la autoridad hídrica. No son siquiera sujetos de otras políticas de emergencia porque no tienen las inscripciones que la provincia exige para el acceso a estos fondos de emergencia. Quedan fuera por situación de informalidad, por deuda o por falta de rentabilidad.

De manera similar a los pequeños productores de la zona irrigada (columna 3, cuadro 2), son los organismos nacionales (en ocasiones a su vez financiados por organismos internacionales) los que llegan a ellos para asistirlos pero solo en temas de producción (aprovechamiento del agua de vertientes a fin de producir pasturas y para promover la producción de forrajes en los puestos en zona de producción caprina; compra de alimento balanceado para poderla trabajar en todo el territorio provincial con la población); o en hábitat y sanidad (proveer instalaciones mínimas para alimentar a viviendas); o relevamiento de información. También reciben asesorías en derechos y fortalecimiento institucional pero realmente poco significa esto para una mejora radical de su condición. En asuntos exclusivos de agua, en general las ayudas no pasan de algunas herramientas básicas en coyunturas en que la sequia ha sido significativa.

Prácticas de apropiación y uso: Las prácticas de apropiación y uso del agua en parte son consecuencia del patrón de prácticas de gestión y también varían según el tamaño de productor y tipo de cultivo. El trabajo de Montaña (2013) desarrolla un análisis exhaustivo de este tipo de acciones:

Los grandes productores irrigados (vitivinicultores, horticultores) (columna 1 del cuadro 2) utilizan agua de pozos (la mitad de ellos, como fuente exclusiva de abastecimiento). Esto también los independiza de los mecanismos de administración del riego, como mencionamos más arriba (Montaña 2012). En ocasiones logran almacenar y/o hacer un nuevo uso de las aguas por la posibilidad de construir infraestructura propia. Tienen la posibilidad de reubicar sus proyectos por elección según suelo, clima y agua (en la cuenca cada vez más alto, Montaña 2013). Están en condiciones de invertir en riego y conocimientos, por lo que pueden realizar planificaciones de largo plazo sobre sus actividades productivas.

Los medianos y pequeños productores irrigados (columna 2 del cuadro 2) tienen acceso al agua de pozos pero sólo en forma limitada por el costo que ello genera. Como explica la autora en su texto, la interacción con las instancias intermedias de gestión del agua junto a las relaciones que establecen con los gobiernos municipales los habilita a integrar un conjunto de redes de productores/instituciones, densa pero con escasos beneficios concretos para su producción (Montaña 2013).

La falta de agua necesaria para alcanzar niveles óptimos de producción junto a los eventos climatológicos adversos los lleva a adoptar una serie de prácticas que no son elegidas sino que en ocasiones son las únicas opciones para mantener su posición productiva. Son prácticas que se vuelven ineludibles para ellos y no tanto adaptaciones a las nuevas situaciones ya que no son deliberadas. El recorte de superficies cultivadas (de manera más contundente en los cultivos hortícolas anuales y más paulatina en cultivos permanentes como los de frutales) marcan una disminución de las expectativas de los actores en producción y calidad. Otro tipo de consecuencias son las que suceden en el caso del olivo, por ejemplo, en que la disminución de la producción es indirecta y provocada no por la cantidad de tierra cultivada sino por la restricción de agua. Algunos han llegado al extremo de tener que cambiar de actividad (o han dejado de sembrar, o han ido vendiendo sus terrenos para inclinarse a otros sectores como el turismo o las artesanías, pero de manera muy informal, o bien se mantienen en la producción pero se pasan a otros cultivos menos demandantes de agua, aunque, a la vez, mucho menos rentables (por ejemplo, las hierbas aromáticas).

Dinámica y desempeño: los aspectos de la estructura formal y las prácticas de gestión y apropiación 
nos aportan información valiosa para comenzar a comprender la lógica de funcionamiento del orden hídrico. Pensemos ahora por un momento en los patrones en que esas estructuras formales se articulan con las prácticas. Del caso que estamos analizando, es posible advertir que lejos está de ser un sistema policéntrico con varias unidades de gobierno, con diferentes propósitos, organización y ubicación espacial. Tampoco existe una fluida interacción entre las instituciones formales. Se trata, por el contrario, de un sistema altamente centralizado donde el poder está concentrado en un nivel único y superior. Por otro lado, existe una serie de instituciones satélites de la central, que sólo tienen incidencia indirecta sobre el recurso hídrico pero que, al parecer, son las únicas instituciones al alcance de los actores pequeños y medianos. Entre estas organizaciones están altamente conectadas para llegar a los productores pero lo que ellas pueden asistir para mejorar el acceso al agua es limitado. En un circuito diferente encontramos a los actores productivos más grandes y poderosos en relación directa con la autoridad hídrica. Esta última no sólo es autárquica sino aislada. Con este esquema, la dinámica institucional sostiene y reproduce unas relaciones de desigual acceso a las posibilidades de acceder al agua y negociar lo que no se tiene. Ante esto es muy difícil pensar en un acceso equitativo al recurso.

Instituciones informales: la forma en que un sector de la producción local (los grandes productores vitícolas) tiene relación directa y contactos estrechos con las autoridades hídricas mientras que otros se ven afectados porque no logran acceder a los volúmenes de agua que sus actividades requieren, constituye un conjunto de reglas no escritas pero que son las que en definitiva terminan rigiendo el orden hídrico. El carácter relativamente establecido de estas normas da forma a las instituciones informales. Estas reglas que en definitiva marcan cómo deben actuar los actores se acomodan a las estructuras formales y conviven, creando o restringiendo los incentivos para actuar de manera diferente a lo que el espíritu de la norma establece, sin violarla directamente. En el caso de los pequeños productores, la falta de incentivos para acceder a un trato directo con la autoridad hídrica los lleva a que ni siquiera lo intenten y permanezcan así en un circuito institucional secundario (en el sentido que no son entidades que tengan injerencia directa sobre el agua), por más que estén habilitados por la norma formal. Un ejemplo de este tipo de instituciones es el reparto de volúmenes excedentes de agua producidos por el abandono de muchas fincas (Martín 2008). Esta reasignación se realiza según el criterio del agente encargado de entregarla.

Esta dinámica está sostenida por las cosmovisiones de los actores. No sólo los productores sino los mismos funcionarios representantes de las instituciones satélites al agua. Los constreñimientos que los pequeños y medianos productores tienen para acceder al Departamento General de Irrigación es una creencia ampliamente compartida y alimenta la inercia al cambio.

\section{RECAPITULACIÓN}

Los avances de investigación que expusimos en estas páginas tuvieron el propósito de mostrar una manera diferente a la dominante de indagar la relación entre las instituciones del agua y los actores. Estamos convencidos de que para comenzar a resolver la distancia y desconexión entre las propuestas de gestión hídrica y las problemáticas que sufren los actores usuarios del agua es fundamental desarrollar un enfoque que mire de cerca no solo los aspectos más formales de los regímenes sino a los actores y sus interacciones.

El breve análisis que aquí presentamos es un claro indicio de que los actores locales que reciben el agua no pueden ser considerados desde la política - ni la investigación- como si fueran un solo actor homogéneo. Por más que la ley y la autoridad hídrica sean las mismas para todos, las apropiaciones que de ellas hacen los actores son muy diferentes según su posición. De igual forma, lo que las instituciones «ofrecen» en la práctica a cada sector es diferente.

El acercamiento a las interacciones instituciones-usuarios nos habla de un patrón en que se desarrolla una dinámica de dos circuitos con dos características diferentes. Por un lado, un esquema centralizado para las decisiones estrictamente hídricas que interacciona con los productores más poderosos (los grandes empresarios de la industria vitícola). Por otro lado, un esquema de coordinación horizontal entre diferentes entidades institucionales -no específicas del agua sino de ambiente y producción- ni de la jurisdicción o el nivel de gobierno al 
que le compete la autoridad jurídica del agua -no provinciales sino municipales y nacionales-.

La consecuencia de este esquema es la reproducción de las desigualdades de las condiciones y la escasa posibilidad de mejorar su situación productiva $\mathrm{y}$, en consecuencia, socioeconómica. Este tipo de procesos no salen fácilmente a la luz con las miradas dominantes. Un programa de investigación que se mantenga en esta posición y que desde allí genere acciones destinadas a la gestión del recurso hídrico, creemos, será útil para inspirar políticas y programas próximos a las problemáticas locales. No es una garantía, pero sí una forma de acercarnos a un grado aceptable de consistencia entre las reformas propuestas y las condiciones sociales, económicas y políticas.

\section{FUENTES DE CONSULTA}

Aguilar, Luis, 2007, «El aporte de la política pública y de la nueva gestión pública a la gobernanza», Revista del CLAD Reforma y Democracia, n. 39, Caracas, pp. 1-15.

Birkenholtz, Trevor, 2011, «Network political ecology: Method and theory in climate change vulnerability and adaptation research», Progress Human Geography, v. 36, pp. 295-315.

Metha, Lyla, Melissa Leach, Peter Newell, Ian Scoones, Kalyanakrishnan Siravaramakrishnan y Sally-Anne Way, 1999, «Exploring understandings of institutions and uncertainty: new directions in natural resource management», IDS Discussion Paper 372, Environment group, Institute of Development Studies, pp.1-48.
Martín, Facundo, 2008, «Agua y modelo productivo. Innovaciones tecnológicas e impactos territoriales en el sistema agroalimentario de Mendoza», Estudios socioterritoriales. Revista de geografía, n. 7, pp. 26-45.

Montaña, Elma, 2013, Escenarios de cambio ambiental global, escenarios de pobreza rural. Buenos Aires: CLACSO.

__, 2012, «Vulnerabilidades pasadas y presentes de los productores agrícolas y ganaderos de la cuenca de Mendoza», en Vulnerabilidad al cambio ambiental global. Desafíos para la adaptación en las cuencas de Elqui y Mendoza, editado por Salas, Sonia, et al., Instituto Interamericano para la Investigación del Cambio Global, La Serena, pp. 59-74.

Mosse, David, 2006, «Collective action, common property and social capital in South India: an anthropological commentary» Economic Development and Cultural Change, v. 54, n. 3, pp. 695-724.

Nuijten, Monique, 2005, «Power in Practice: A Force Field Approach to Natural Resource Management», The Journal of Transdisciplinary Environmental Studies, v. 4, n. 2, pp. 1-14.

Ostrom Elinor y Edella Schlager, 1992, «Property rights regimes and natural resources: a conceptual analysis», Lands and Economics, v. 68, n. 3, pp. 249-262

Ostrom, Elinor, 2008, «Institutions and the environment» en The economic analysis of institutions. Blackwell Publishing. Oxford, pp. 24-31.
Fecha de recepción: 5 de septiembre de 2013. Fecha de aceptación: 25 de noviembre de 2013.

P. C. Mussetta • Orden hídrico: prácticas e instituciones

en Mendoza, Argentina 